\title{
O021. Abnormal connectivity within executive resting-state network in migraine with aura
}

\author{
Antonio Russo ${ }^{1,2,3^{*}}$, Francesca Conte ${ }^{1}$, Laura Marcuccio ${ }^{1}$, Fabrizio Esposito ${ }^{4}$, Alfonso Giordano ${ }^{1,2}$, \\ Manuela De Stefano ${ }^{1}$, Mario Cirillo ${ }^{5}$, Alessandro Tessitore ${ }^{1}$, Gioacchino Tedeschi ${ }^{1,2,3}$ \\ From Abstracts from the 1st Joint ANIRCEF-SISC Congress \\ Rome, Italy. 29-31 October 2015
}

\section{Background}

Despite the fact that the clinical features of migraine are well described, the relationship between migraine and cognitive performance is still poorly understood. Indeed, some authors have reported the presence of cognitive deficits in patients with migraine without aura (MwoA) and with aura (MwA) whereas others have not confirmed these findings. Although neuropsychological studies in migraine are not conclusive, the most likely pattern of neuropsychological impairment would relate to the cognitive domain of executive functions (EF) [1]. Recent imaging studies have shown a significant functional connectivity decrease within the fronto-parietal networks (FPN), known to be associated with EF, in patients with MwoA in absence of significant executive dysfunction [2].

\section{Objective}

To further explore FPN functional connectivity in patients with MwA and patients with MwoA, in the interictal period.

\section{Methods}

Using resting-state functional magnetic resonance imaging (RS-fMRI), we compared functional connectivity within the FPN in 20 patients with MwA, versus 20 sex- and agematched healthy controls ( $\mathrm{HC}$ ). To examine the specificity of any observed differences in FPN functional connectivity between patients and $\mathrm{HC}$, we further studied 20 age- and sex-matched patients with MwoA. Furthermore, we assessed the correlation between functional connectivity within FPN and EF in both migraine groups. Finally, we

\footnotetext{
* Correspondence: dottor.russo@gmail.com

'Department of Medical, Surgical, Neurological, Metabolic and Aging

Sciences, Second University of Naples, Naples, Italy

Full list of author information is available at the end of the article
}

used voxel-based morphometry to assess whether between-group differences in functional connectivity were dependent on structural differences.

\section{Results}

Neuropsychological data revealed no significant executive dysfunction in both migraine groups compared to HC. RS-fMRI showed that both MwA and MwoA patients, compared to $\mathrm{HC}$, had a significant functional connectivity decrease within the right FPN and specifically in the middle frontal gyrus and the dorsal anterior cingulate cortex. There were no structural differences between the three groups.

\section{Conclusions}

Our data demonstrate that, even in the absence of clinically evident EF deficits, MwA and MwoA are associated with reduced FPN functional connectivity. We suggest that disrupted FPN functional connectivity might be only a part of a complex cascade that terminates in a migraine attack. In this context, FPN abnormalities may be the neuronal substrate on which biological, genetic and environmental factors could induce, and in turn correlate with, migraine attacks mostly characterized by high pain intensity in patients with MwoA and aura phenomenon in patients with MwA. In other terms, observed FPN connectivity changes may represent a migraine biomarker, probably related to well-known maladaptive stress response in migraine patients.

Written informed consent to publish was obtained from the patient(s).

\section{Authors' details}

${ }^{1}$ Department of Medical, Surgical, Neurological, Metabolic and Aging Sciences, Second University of Naples, Naples, Italy. ${ }^{2} \mathrm{MRI}$ Research Center SUN-FISM, Second University of Naples, Naples, Italy. ${ }^{3}$ Institute for Diagnosis 
and Care "Hermitage Capodimonte", Naples, Italy. ${ }^{4}$ Department of Medicine and Surgery, University of Salerno, Baronissi (SA), Italy. ${ }^{5}$ Neuroradiology Unit,

Department of Clinical and Experimental Medicine and Surgery, Second

University of Naples, Naples, Italy.

Published: 28 September 2015

\section{References}

1. Schmitz N, Arkink EB, Mulder M, Rubia K, Admiraal-Behloul F,

Schoonman GG, et al: Frontal lobe structure and executive function in migraine patients. Neurosci Let 2008, 440(2):92-96.

2. Russo A, Tessitore A, Giordano A, Corbo D, Marcuccio L, De Stefano M, et al: Executive resting-state network connectivity in migraine without aura. Cephalalgia 2012, 32(14):1041-1048.

doi:10.1186/1129-2377-16-S1-A156

Cite this article as: Russo et al:: O021. Abnormal connectivity within executive resting-state network in migraine with aura. The Journal of Headache and Pain 2015 16(Suppl 1):A156.

\section{Submit your manuscript to a SpringerOpen ${ }^{\mathcal{O}}$ journal and benefit from:}

- Convenient online submission

- Rigorous peer review

- Immediate publication on acceptance

- Open access: articles freely available online

- High visibility within the field

- Retaining the copyright to your article

Submit your next manuscript at $\gg$ springeropen.com 